\title{
STEPHEN DAVIES
}

\section{Assaults and threats on psychiatrists}

\section{AIMS AND METHOD}

To determine the annual rates of assaults and threats to psychiatrists, describing the situations and staff involved, using a retrospective postal questionnaire of 139 doctors working in South Wales.

\section{RESULTS}

Over the year, $17 \%$ of respondents reported one or more assaults (of these, $42 \%$ were assaulted more than once) and $32 \%$ reported one or more threats. The most junior senior house officers (SHOs) were significantly more likely to have experienced an incident, regardless of the individual's gender or attendance at a course in managing aggression. Most assaults $(61 \%)$ were committed by patients from general adult psychiatry, and half occurred during urgent assessments. Eighteen (58\%) of the assailants were known to have previously assaulted a member of staff, and this information was known to the doctor before the assault for $16(88 \%)$. Five (16\%) of the assailants had been drinking alcohol prior to the assault. Twenty-nine $(78 \%)$ of the assaults were documented in the case notes and 6 $(19 \%)$ were reported to management.

\section{CLINICAL IMPLICATIONS}

Some staff (in particular, inexperienced SHOs) are at greater risk, and efforts should be made to identify and help these individuals and/or grades to deal with aggression. All psychiatric staff should be trained to manage violence. Staff should be encouraged to report incidents to management, so that employers can identify and address problem areas.
Previous reports show that $25--60 \%$ of psychiatrists have been assaulted while at work (Fottrell, 1980; Health Services Advisory Committee, 1987; Madden et al, 1976; O'Sullivan \& Meagher, 1998). A thorough review of the literature by the Royal College of Psychiatrists (1998) concluded that there was weak evidence that training and experience in coping with aggression reduced injuries; only a suggestion that good leadership and avoidance of overcrowding can reduce violence; and a lack of consensus on what items are useful for short-term prediction of violence. However, another recent literature review concluded that certain risk factors could be identified (Shaw, 2000). These include demographic factors (being male, young and from a poor socio-economic background), symptoms (acute psychotic symptoms, specifically certain types of delusion), previous violence, substance misuse, purchase of a weapon and the making of threats to identifiable victims.

This study sets out to determine the rates of threats and assaults against psychiatrists in one region of the UK, and to describe in what situations and to which staff these incidents occur, examining the implications for training

\section{Method}

In 1997, all doctors working in psychiatry in South Wales were identified from records held by hospital postgraduate and personnel departments. A questionnaire was posted to those identified, asking the individual's gender, grade, sub-speciality, whether the individual had been assaulted or threatened by a psychiatric patient during the previous 12 months and if so, whether this had been documented in the case notes or reported to management on incident forms. Assaults were defined as being 'hit, struck with an object, shaken, pushed, throttled, etc.'. Threats were defined as patients expressing 'specific intentions to physically harm you (i.e. either directly, or by making threats reliably conveyed to you by colleagues) as a separate incident to any assault'. Those indicating that they had been assaulted were asked to complete an additional questionnaire at the same time, giving details of the circumstances and outcome of the assault. All replies were anonymous. The questionnaires were sent out during the final month of the senior house officer (SHO) rotation. A stamped addressed envelope was provided for reply.

\section{Results}

Of the 198 doctors identified as working in psychiatry, 139 returned responses (70\%), of whom 83 (60\%) were male and 56 (40\%) were female. 
Table 1. Frequency of assaults and threats reported by respondents $(n=139)$

original papers
Incident Number of incidents Number of respondents (\%)

$\begin{array}{lrr}\text { Assault } & 0 & 115(83 \%) \\ & 1 & 14(10 \%) \\ & 2 & 8(6 \%) \\ \text { Threat } & 3 & 1(1 \%) \\ & 4 & 1(1 \%) \\ & 0 & 94(68 \%) \\ & 1 & 26(19 \%) \\ & 2 & 14(10 \%) \\ & 3 & 3(2 \%) \\ & 4 & 2(1 \%)\end{array}$

\section{Assaults}

Twenty-four (17\%) respondents had been assaulted at least once during the previous 12 months. Of these, 14 $(58 \%)$ were male and $10(42 \%)$ were female. A total of 37 assaults were reported during the study period. Of the respondents reporting an assault, $10(42 \%)$ had been assaulted more than once (Table 1).

\section{Threats}

Forty-five (32\%) of the 139 respondents had been threatened at least once during the previous 12 months. Of these, $32(71 \%)$ were male and $13(29 \%)$ were female. A total of 71 threats were reported during the study period. Of the respondents reporting a threat 19 (42\%) had been threatened more than once (Table 1).

Table 2 shows the number of assaults and threats according to the grade of doctor. Thirteen of the SHOs had worked in psychiatry for less than 12 months, so the number of assaults and threats per year were calculated by extrapolation from the number of months worked. The rate per person per year according to grade has been calculated, with $95 \% \mathrm{Cls}$.

Twenty-nine $(78 \%)$ of the assaults were documented in the case notes and $6(19 \%)$ were reported to management. For threats, the figures were $34(48 \%)$ and $8(11 \%)$, respectively.

Of the respondents, 67 (48\%) had attended a course on dealing with aggressive patients, which 58 (87\%) found useful. Among the 67 who had attended a course, there were 19 assaults, compared to 18 among the 72 who had not. These differences were not statistically significant $\left(\chi^{2}=0.144, P>0.1\right)$.

\section{Assaults: additional information}

Respondents were then asked to provide additional information on the circumstances of each individual assault. Information was provided for 31 (84\%) of the 37 assaults. Of the assaults on female doctors, nine were by female patients and two by male patients. Male doctors were assaulted five times by female patients and 15 times by male patients. This suggests that doctors were more likely to be assaulted by patients of their own gender, although the differences were not significant $\left(\chi^{2}=0.19\right.$, $P>0.1$.

The doctors reported that 18 (58\%) of the assailants were known to have previously assaulted a member of staff, and that in $16(88 \%)$ of these cases this information was known to them before the assault. Five (16\%) of the assailants were reported to have been drinking alcohol prior to the assault.

The assaults tended to occur in circumstances where the patient was seen urgently: seven $(23 \%)$ involved patients referred for urgent assessment between 9.00 a.m. and 5.00 p.m. weekdays, eight (26\%) for urgent assessments outside these hours and four (13\%) following requests for urgent reviews of an in-patient. This compares to only one (3\%) during routine outpatient assessments and follow-ups. Other situations included domiciliary visits (five, 16\%), routine in-patient reviews (three, 10\%) and casual contact, e.g. in a day room or corridor (three, 10\%).

Only four assaults (13\%) occurred while the doctor was alone with the patient. The remainder occurred in the company of a nurse $(74 \%)$ or doctor $(13 \%)$, and none in the presence only of a non-staff member.

The majority of assaults 19 (61\%) were carried out by patients from general adult psychiatry, with five (16\%) from forensic psychiatry, three $(10 \%)$ in learning disability, two $(6.5 \%)$ from child psychiatry, one (3\%) from substance misuse and one (3\%) unclassified. No assaults were reported by patients from rehabilitation or old age psychiatry.

The physical consequences of the assaults were mainly non-serious. Only three $(10 \%)$ resulted in time away from clinical duties, and in all three cases this

Table 2. Frequency of assaults and threats experienced by doctors of various grades

\begin{tabular}{|c|c|c|c|c|c|}
\hline Grade & Respondents ( $n$ ) & Assaults ( $n)$ & $\begin{array}{l}\text { Assaults per person } \\
\text { per year }(95 \% \mathrm{Cl})\end{array}$ & Threats $(n)$ & $\begin{array}{l}\text { Threats per person per } \\
\text { year }(95 \% \mathrm{Cl})\end{array}$ \\
\hline Senior house officer ( $<$ year) & 13 & 5 & $0.77(0.43-1.00)$ & 7 & $1.12(0.84-1.40)$ \\
\hline Senior house officer ( $>$ year) & 43 & 11 & $0.26(0.14-0.38)$ & 26 & $0.60(0.45-0.75)$ \\
\hline Senior/specialist registrar & 17 & 8 & $0.47(0.23-0.71)$ & 9 & $0.53(0.29-0.77)$ \\
\hline Consultant & 59 & 12 & $0.20(0.10-0.30)$ & 28 & $0.47(0.33-0.61)$ \\
\hline Staff grade & 4 & 1 & $0.25(0.00-0.67)$ & 1 & $0.25(0.00-0.67)$ \\
\hline Other & 3 & 0 & 0 & 0 & 0 \\
\hline Total & 139 & 37 & 0.27 & 71 & 0.51 \\
\hline
\end{tabular}


was for less than 30 minutes. None required any physical treatment.

\section{Discussion}

Owing to the retrospective nature of this study, it is likely to suffer from selective recall and response bias.

The number of assaults and threats reported is smaller than in previous studies (Fottrell, 1980; Health Services Advisory Committee, 1987; Madden et al, 1976; O'Sullivan, 1998). The majority of those responding reported no incidents. However, nearly half of those assaulted reported more than one assault. Also, the same individual who reported the maximum number of assaults also reported the maximum number of threats. Therefore, there may be individuals who are at particular risk of assault because of the nature of their work or their individual practices. There is a need to identify such individuals and determine how they may reduce the risk of future incidents in a manner that avoids victim blame.

Overall, the results suggest a decrease in risk of threat and assault as seniority increases (with the exception of specialist and senior registrars, who reported more assaults per person per year than the more experienced SHOs). However, when 95\% Cls were calculated, the only group revealing a significantly higher rate of assault was the more inexperienced SHOs. Similarly, with respect to threats, the newer SHOs reported a significantly higher rate than all other grades. This is unsurprising, but has not been reported elsewhere. One explanation might be that the juniors were seeing more acutely disturbed patients than were the consultants (in casualty, for example). However, this would not explain the trend to increased safety in the more experienced SHOs, performing duties similar to those of newer SHOs. It suggests that the overall trend to increased safety with seniority is because of experience, rather than the performance of different duties, and highlights the need for education on the management of violence early in training. The observation that most assaults involved patients from general adult psychiatry is consistent with this, as most trainees work in this area first, and almost all work on-call in this field. The study shows that patients referred for urgent assessment are the source of over half of the assaults sustained. Anecdotally, this is an area in which trainees often work unsupervised, and perhaps more observation (by and of trainees) would be useful.

However, the absence of an association between being assaulted or threatened and having attended a course in managing violence would not seem to support these recommendations. It may be because the study failed to differentiate between different types of course, or ask how recently the course had been attended. The courses cannot therefore be dismissed, and were reported as being useful by most respondents. The Royal College of Psychiatrists (1998) has suggested that attendance at such courses may reduce the severity rather than number of incidents. Also, there is more to safety awareness training than attending a course (Chubb,
1997). The following suggest that staff were acting on available information to reduce risk: (a) the lack of any serious physical injury (in contrast to previous reports with rates of $11-12 \%$ (Health Services Advisory Committee, 1987; O'Sullivan \& Meagher, 1998)); (b) the fact that when assaults did occur they tended to be in the presence of another person; and (c) the finding that the patients' previous histories of assault were known to most of the doctors.

The study did not ask about the psychological consequences of the assaults (partly because of the lack of a very brief objective means of rating this) or what support was offered. Although $90 \%$ of those assaulted did not take any time away from clinical duties, this cannot be taken to indicate that the incidents lacked emotional or psychological impact.

The Health and Safety at Work Act places a responsibility on employers to identify and address hazards in the workplace. Few assaults and even fewer threats were reported to management, making this process difficult. The study did not explore possible reasons for this, although the fact that the majority of assaults (but not threats) were documented in the case notes suggests a lack of awareness of this notification process (or of its purpose), rather than an underestimation of the importance of the actual assault, or even guilt on the part of the doctor.

The tendency for doctors to be assaulted by patients of their own gender, although not statistically significant, is intriguing, but it requires replication before we speculate on its possible causes.

In conclusion, the scope for further reduction of incidents and the need for training is suggested by several observations. While there may be many reasons for higher reporting of incidents by some individuals, it would be sensible to propose that some sort of debriefing facility, in a blame-free environment, be made available, both for emotional support of the victim and to aim to reduce future incidents. Finally, there is a need for further research on the behaviour that distinguishes experienced from inexperienced psychiatrists, and how the latter can be most effectively helped.

\section{References}

CHUBB, H. (1997) Safety awareness among junior psychiatrists and provisions for their safety in the workplace. Psychiatric Bulletin, 21, 80 83.

FOTTRELL, E. (1980) A study of violent behaviour among patients in psychiatric hospitals. British Journal of Psychiatry, 136, 216-221.

HEALTH SERVICES ADVISORY

COMMITTEE (1987) Violence to Staff in the Health Services. London: Health and Safety Commission.

MADDEN, D. J., LION, J. R. \& PENNA, M. W. (1976) Assaults on psychiatrists

by patients. American Journal of Psychiatry, 133, 422-425.

O'SULLIVAN, M. \& MEAGHER, D. (1998) Assaults on psychiatrists - a three year retrospective study. Irish Journal of Psychiatric Medicine, 15, 54-57.

ROYAL COLLEGE OF PSYCHIATRISTS (1998) Management of Imminent Violence. Occasional Paper OP41.

London: Royal College of Psychiatrists.

SHAW, J. (2000) Assessing the risk of violence in patients. British Medical Journal, 320, 1088-1089.

Stephen Davies Specialist Registrar, Whitchurch Hospital, Cardiff CF4 7XB 